\title{
Los ingresos tributarios de las haciendas locales y la crisis económica
}

\author{
$M^{a}$ José Portillo Navarro
}

RESUMEN: La crisis económica ha afectado a la actividad económica en España y, por tanto, a los municipios españoles, existiendo una serie de variables que han podido incidir en la gestión de la situación presupuestaria local y, entre ellas, se encuentra la evolución de los tributos locales. Por ello, en este trabajo, nos planteamos como objetivo analizar las diferencias entre los municipios, agrupados según tamaño, durante los años posteriores al inicio de la crisis, en términos de recaudación por habitante, eficacia de la gestión recaudatoria y esfuerzo fiscal. Los resultados muestran las diferencias en la evolución de los ingresos fiscales por tamaño de población y sugieren que los municipios tienen margen para aumentar sus recursos tributarios mediante la mejora de la eficacia de la gestión recaudatoria, teniendo todavía recorrido para incrementar su esfuerzo fiscal.

PALABRAS CLAVE: Crisis económica, recaudación tributaria, impuestos locales, indicadores, presión fiscal.

CLAVES ECONLIT: H20, H70, K34, M40.

Cómo citar este artículo / How to cite this article: PORTILLO, M.J. (2018): "Los ingresos tributarios de las haciendas locales y la crisis económica", CIRIEC-España, Revista de Economía Pública, Social y Cooperativa, 92, 253-278.

Correspondencia: Ma José Portillo Navarro, Profesora Titular, Universidad de Murcia. E-mail: portillo@um.es. 


\section{EXPANDED ABSTRACT}

\section{The local taxes revenues and economic crisis}

Local entities had needed to overcome economic crisis as the rest of the public and private organism. Council made expenditures that did not correspond to them as they were not belonging to them competencies.in time of big public incomes -They come mainly from the boom of the urban activity in Spain- When the crisis in the real estate sector came, revenues were reduced and a reordering of the provision of public services was made between the Autonomous Communities and the local councils through different laws to avoid duplication of the provision of public services between administrations, the structure organizational structure of local authorities were razionalizate with an unequal result.

However the evolution of budgetary figures has not be the same in all the councils. Some municipalities have suffered complicated situations, while others have not, between some factors, It is related with the local budget situation before the crisis starts - that is the bigger or lower prior sanitation of public accounts- and afterward with the management of the crisis and the size of the municipality, since the cost of providing the municipal public service is related among other factors, to the greater or lesser population of the municipality and the obligation or not to provide these services. (Suárez, 2007; Tránchez, et. al, 2011).

The size of the municipalities is an important element in the current configuration of the councils. One of the biggest problem of the local sector is the existence of a large number of small municipalities. In fact, 83,8 percent of the Spanish municipalities have less than 5.000 inhabitants and they group 12,7 percent of the population, while 10.3 percent of the inhabitants are in two municipalities larger than 1.000 .000 inhabitants (Madrid and Barcelona). Altogether, 95,8 percent of the Spanish municipalities have less than 20.000 inhabitants and they group 31,9 percent of the Spanish population. Another element of interest is the prevailing heterogeneity among all (Angoitia, 2005) related to the competences attributed to them by the "Law of local regime bases", in terms of the provision of services which changes according to their size.

In order to face the local expenditure, councils need incomes, among them, the tax revenues with great importance in the municipalities, so a greater or lesser collection affects their autonomy to develop their fiscal policy. In addition, as the local authority has more own resources, it would not have to ask for debt in order to be able to implement its public expenditure. (Guillamón, Benito and 
Bastida, 2011; Pedraja, 2011; Suárez y Fernández, 2012; Balaguer-Coll, Prior y Tortosa- Ausina, 2016).

Thus, in this paper, we lay out to analyze the differences between the municipalities, grouped by size, during the years following the onset of the crisis, analyzing several indicators: revenue per capita, the effectiveness of tax collection and tax effort.

It is necessary to analyze the evolution of tax revenues per inhabitant or local tax pressure defined as the relationship between taxes and the number of residents. (Zafra and López, 2006; Benito et al, 2010) This indicator has increased for all Spanish municipalities from the beginning to the end of the period 2006-2014 for all the segments of population analyzed, except for municipalities with a population between 5.000 and 20.000 inhabitants, which may be related to the evolution of local taxes. Tax on Real Estate, IBI; Tax on Vehicles of Mechanical Traction, IVTM, Tax on Economic Activities, IAE, Tax on Constructions, Installations and Works, ICIO, and Tax on Increase in the Value of Urban Land, IIVTNU.

If we consider the type of tax, we see how the municipalities over 1.000 .000 inhabitants are the ones that have increased the income from the Real Estate Tax, which is the tax that brings the highest collection to the city councils.

Effectiveness of the collection management is a good indicator to know the compliance of the local budget and it has been analyzed in this paper. If we focus on taxes, IBI, IAE and IVTM are those that present a greater efficiency of the collection management, that probably will be related to the fact of being managed through registers about which the municipality has all the data of the taxpayer. After the analysis, it is evident the need to improve the efficiency of the collection management by all municipalities, since the councils have reduced this indicator from the beginning to the end of the period for all taxes, mainly in those of population between 500.000 and 1.000 .000 inhabitants, it contrasts with the fact that the municipalities with the largest population have more material and human resources to carry out their tax management.

Fiscal effort has also been calculated on this paper as we are considering the definiton of it as the relationship between the real collection and the potential collection and, for this, we have had to calculate the potential collection for each of the taxes, since this variable it is not available.

The obtained results show that from 2006 to 2014 the municipalities have reduced their fiscal effort in all taxes, which contrasts with the financial needs of this period. Therefore, Spanish municipalities can still increase their collection in a notable way in some taxes, such as the IBI or the $I A E$. In the case of the IBI, despite the increase in rates in the recent years, there is still being an important margin for the increase in collection. 
By sections of population, there are important differences. Thus, municipalities with more than 100.000 inhabitants have increased their tax effort in almost all taxes, although it is necessary to consider that they are also those with the highest income. In any case, we can not find a pattern of behavior of all municipalities, because while the ones with population between 50.000 and 100.000 inhabitants have increased their tax effort in the IAE by 8,9 points from the beginning to the end of this period, the municipalities of less than 5.000 inhabitants have reduced their fiscal effort. And the same happens in other cases and taxes.

However, the fact that Spanish municipalities can increase their fiscal effort, mainly in relation to the $\mid \mathrm{BI}$ and to the rest of taxes, does not necessarily imply the need to increase their collection (Bahl, 1971, Vallés and Zárate, 2013). In addition, we must consider that some municipalities may decide to demand less taxes because they can decide the implementation of a more restrictive public expenditure policy (Cheliah et al, 1975, Vallés and Zárate, 2013).

One aspect that may have affected is the increase in the last years of the tax rate of the $|\mathrm{B}|$, which affects the potential collection and the increase thereof, assuming an increase in the denominator of the defined fiscal effort, which may have been its consequences in the fiscal effort figures obtained.

The results obtained in this paper show that Spanish municipalities in order to face the economic crisis generally have chosen to increase tax resources, thus increasing their financial autonomy, mainly through the $\mid \mathrm{BI}$, what is the main figure of local taxation, being the municipalities over $1,000,000$ inhabitants which have increased tax revenues per capita to a greater extent from the beginning to the end of the period.

The problems arising from the rigidity of local taxes have undoubtedly contributed to the current situation of the Local Treasury, which should be subject to modification and meet criteria that would lead to a greater elasticity of this type of taxation, which could mean an important contribution to the achievement of greater municipal self-financing.

The limitations of this paper came from the availability of data for the calculation of fiscal effort. However, we believe it is necessary to progress in this indicator for future research.

In any case, this line of this paper may continue to be the subject of numerous studies that may deepen the relationship between tax collection and other indicators that may add new perspectives to the study of the local tax situation, with new lines of reform that may be arised in the future. 
However, the results obtained can be of great interest to the local manager and decision-making in their municipality, considering the situation of the municipalities that are in the same stretch of population. These results can be used to find out solutions to the problems analyzed in this paper.

KEYWORDS: Economic crisis, tax collection, local taxes, indicators, tax pressure. 


\section{Introducción 1}

El análisis de la recaudación local se ha abordado en numerosas ocasiones, (Castells, 2002; Poveda y Sánchez, 2002; Suárez, 2005, por citar algunos ejemplos) mostrando el raquitismo crónico de la Hacienda Local y su dependencia de los recursos provenientes de otras instancias supramunicipales, tales como las Comunidades Autónomas o el Estado. Ello ha supuesto que los problemas relacionados con la Hacienda local, sus competencias y su financiación hayan sido objeto de modificaciones legislativas importantes en los últimos años, derivados principalmente de la crisis económica y sus consecuencias. Así, la reforma del art. 135 de la Constitución española, que supuso la consagración de la estabilidad presupuestaria, tuvo como consecuencia la aprobación en 2012 de la Ley de estabilidad presupuestaria y sostenibilidad financiera con importantes repercusiones en la esfera local.

Además, con el transcurso del tiempo y el desarrollo del Estado autonómico, se habían producido diversos problemas generados como consecuencia de la escasa definición, en algunos casos, de las competencias para la prestación de servicios, lo cual derivó en la asunción por parte de las entidades locales de la prestación de algunos de estos servicios sin contar con financiación especial del Estado 0 de las Comunidades autónomas, lo cual supuso el crecimiento de los gastos impropios o no obligatorios, (Suárez, 2007; Tránchez et al, 2011), que llegaron a suponer, según algunos estudios, alrededor de un tercio de los gastos municipales, superando incluso, en algunos casos, esta cantidad.

Esta necesidad de mejorar la definición de las competencias que debían asumir los municipios supuso la aprobación de la Ley 27/2013, de 27 de diciembre, de racionalización y sostenibilidad de la Administración Local, la cual pretendía clarificar las competencias para evitar la duplicidad de las mismas entre Administraciones, racionalizando la estructura organizativa de los entes locales y avanzando en el principio de "una Administración, una competencia» para evitar los solapamientos que en algunos casos se producían. Sin embargo, esta Ley no se aprobó con el necesario consenso para una norma de este calado, por lo que ha sido ampliamente cuestionada por diferentes sectores relacionados con las entidades locales y se ha reducido su efectividad, con la aprobación de diversas normativas autonómicas que han limitado, en la práctica, su aplicación (Forcadell, 2015), además de los recursos de inconstitucionalidad que se han planteado sobre esta norma.

En este contexto, la situación de las Haciendas Locales sigue necesitando profundas reformas, principalmente, en lo referido a la financiación y modificaciones sustanciales en los impuestos locales, una realidad ya reclamada desde hace años que se repite en el tiempo (Poveda y Sánchez, 2002; 
Suárez, 2005; Pedraja y Suárez 2011), pues los efectos derivados de la normativa aplicable son fundamentales (Suárez, 2011). Además, la evolución de las cifras presupuestarias durante los años de crisis económica no ha sido igual en todos los Ayuntamientos. Así, algunos municipios han estado en situaciones extremadamente complicadas mientras que otros se han encontrado con situaciones menos adversas. Esto tienen que ver, entre otros factores, con la situación presupuestaria local antes de la crisis - es decir, el mayor o menor saneamiento previo de las cuentas públicas -, con la gestión de la crisis y con el tamaño del municipio, pues el coste de la prestación del servicio público municipal está relacionada, entre otros factores, con la mayor o menor población y la obligatoriedad o no en la prestación de los mencionados servicios (Suárez, 2007; Tránchez, et. al, 2011).

En este sentido, el tamaño del municipio es un elemento importante en la configuración actual de las haciendas locales. La existencia de un gran número de municipios muy pequeños es uno de los problemas de los que adolece el sector local. En efecto, el 83,8 por cien de los municipios españoles tienen menos de 5.000 habitantes y agrupan al 12,7 por cien de la población, mientras que el 10,3 por cien de los habitantes se encuentra en dos municipios mayores de 1.000.000 de habitantes (Madrid y Barcelona). En conjunto, el 95,8 por cien de los municipios españoles tienen menos de 20.000 habitantes y agrupan al 31,9 por cien de la población española. Otro elemento de interés es la heterogeneidad imperante entre todos ellos (Angoitia, 2005), relacionada con las competencias que tienen atribuidas por la Ley de bases de régimen local, en cuanto a la prestación de servicios y que varían según el tamaño de los mismos. En la actualidad, los municipios, según el tamaño deben prestar una serie de servicios de manera obligatoria y, si desean prestar otros servicios, pueden hacerlo si se encuentran en situación de equilibrio presupuestario.

Junto a las cuestiones planteadas, debemos considerar que las diferencias entre los sistemas de financiación agravan los problemas de los entes locales, en tanto que la dependencia de la variable población para la obtención de recursos estatales condiciona considerablemente los recursos del ente local. En este sentido, el sistema fiscal local supone una vía importante de obtención de recursos para los municipios. Por ello, el objetivo de este trabajo es analizar algunos de los factores explicativos de la variación de los recursos tributarios locales durante la crisis económica, mediante el análisis de la evolución de la presión fiscal, la eficacia de la gestión recaudatoria y el esfuerzo fiscal, considerando el tamaño municipal como eje sobre el cual poder realizar apreciaciones y conclusiones que nos aporten nuevas perspectivas sobre la situación actual, la superación o no de la crisis económica y las perspectivas de futuro. En este sentido, esperamos que, tras la crisis económica, los Ayuntamientos españoles hayan optado por aumentar la recaudación en términos de recaudación por habitante, hayan mejorado la gestión recaudatoria y hayan incrementado su esfuerzo fiscal para hacer frente a la reducción de ingresos que se produjo tras la crisis económica y la pérdida de ingresos procedentes de otras esferas gubernamentales.

El tema de los ingresos tributarios de las entidades locales tiene una gran trascendencia en el funcionamiento de las mismas, en tanto que una mayor o menor recaudación incide en su autonomía para poder desarrollar su política fiscal. Además, en la medida en la que el ente local disponga de más recur- 
sos propios, no tendrá que acudir al endeudamiento para poder ejecutar su gasto público (Guillamón, Benito y Bastida, 2011; Pedraja, 2011; Suárez y Fernández, 2012, Balaguer-Coll, Prior y TortosaAusina, 2016).

Para ello, este trabajo se estructura del siguiente modo. En primer lugar, realizamos una breve revisión de la financiación local y su evolución durante la crisis económica. En segundo lugar, nos ocupamos de los ingresos fiscales municipales, su variación a lo largo de los últimos años y los factores determinantes de su variación, tales como la recaudación por habitante, la eficacia de la gestión recaudatoria o el esfuerzo fiscal, atendiendo al tamaño del municipio como eje central sobre el cual realizar el análisis. Por último, finalizamos con una serie de conclusiones acerca del trabajo realizado.

\section{El sistema de financiación municipal y la crisis económica}

Antes de iniciar el estudio del sistema de financiación municipal, debemos atender a una cuestión previa y es la relativa al tamaño de los municipios en España. En 2014, en España, existían 8.118 Ayuntamientos, de los cuales, según datos del Ministerio de Hacienda y Administraciones Públicas, el 83,94 por cien tienen menos de 5.000 habitantes y agrupan al 12,58 por cien de la población. Mientras, los municipios de más de 1.000 .000 de habitantes (Madrid y Barcelona) agrupan al 10,19 por cien de los habitantes. Estas diferencias en cuanto a la heterogeneidad de los Ayuntamientos referidas al tamaño, suponen variaciones importantes en cuanto a las variables sociodemográficas (Angoitia, 2005), y una situación que es necesario contemplar, en tanto que la normativa es aplicable, en numerosas ocasiones, a todos ellos, mientras que los medios de unos u otros municipios son bien diferentes, lo cual es una cuestión a resolver sobre la cual se han realizado diferentes propuestas (Caramés, 2005; Suárez et al, 2008; Suárez y Fernández, 2012). Esta situación de gran heterogeneidad, también puede afectar al desarrollo presupuestario, junto con otros factores de interés.

Para proceder al análisis de la financiación municipal, debemos partir de la Ley 39/1988, de 28 de diciembre, Reguladora de las Haciendas Locales - que complementaba la regulación de la Ley la Ley 7/1985, de 2 de abril, Reguladora de las Bases del Régimen Local -, la cual pretendía el desarrollo de la regulación de las Haciendas Locales. En su exposición de motivos se reconoce que la evolución de la Hacienda Local en España es "la crónica de una institución afectada por una insuficiencia financiera endémica" y, desafortunadamente, esta afirmación sigue teniendo vigencia en la actualidad, a pesar de las numerosas reformas realizadas, la de mayor calado en 2002. Con posterioridad, en 2004, se procedió a la realización de un texto refundido que sustituyó a la Ley de Haciendas Locales, el Real Decreto Legislativo 2/2004, de 5 de marzo, por el que se aprueba el texto refundido de la Ley Reguladora de las Haciendas Locales, vigente en la actualidad (en adelante, LRHL). El artículo 59 del 
mencionado precepto enumera el sistema fiscal local y establece que los Ayuntamientos exigirán los siguientes impuestos: Impuesto sobre Bienes Inmuebles (IBI), Impuesto sobre Actividades Económicas (IAE), e Impuesto sobre Vehículos de Tracción Mecánica (IVTM) y podrán establecer y exigir otros impuestos: Impuesto sobre Construcciones, Instalaciones y Obras (ICIO) e Impuesto sobre el Incremento de Valor de los Terrenos de Naturaleza Urbana (IIVTNU). En este sentido, es importante resaltar las potestades de las entidades locales para modificar estos tributos. Tradicionalmente, se ha precisado que los municipios se encuentran en una situación compleja a la hora de establecer nuevos impuestos, pues no tienen potestad normativa para ello, por lo que solamente pueden establecer tasas y contribuciones especiales. Sin embargo, los municipios tienen potestades normativas en cuanto a la modificación de determinados aspectos de los tributos locales - siempre dentro de los márgenes establecidos en la Ley Reguladora de las Haciendas Locales - que van a incidir claramente en sus posibilidades recaudatorias.

En el caso del IBI, las entidades locales podrán ejercer su potestad tributaria en las siguientes materias, fijados ciertos límites en la LRHL: exenciones, tipos impositivos y bonificaciones, lo cual le confiere un margen considerable a la hora de incrementar o reducir la recaudación en un determinado municipio. En el Impuesto sobre Actividades Económicas, las entidades locales podrán ejercer su potestad tributaria a través de la modificación de la cuantía de las cuotas o el establecimiento o no de bonificaciones.

En relación con el IVTM, y a través de la respectiva Ordenanza fiscal, las Entidades locales podrán ejercer su potestad tributaria en materia de modificación de las cuotas municipales y el establecimiento o no de bonificaciones sobre la cuota municipal. Por su parte, los Ayuntamientos que hayan decidido exigir el IIVTNU podrán, en las condiciones que señale la Ordenanza Fiscal respectiva, regular las reducciones en la base imponible, el tipo impositivo (sin superar el 30 por cien) o establecer o no una bonificación regulada en la LRHL, con ciertos límites. Por último, en lo referido al ICIO, Ios Ayuntamientos que hayan decidido exigirlo podrán, en las condiciones que señale la Ordenanza Fiscal respectiva, fijar el tipo de gravamen (siembre que no exceda del 4 por cien) y establecer o no ciertas bonificaciones y deducciones.

Por lo tanto, las potestades establecidas por la Ley reguladora de Haciendas Locales son muy importantes y pueden tener una incidencia relevante en la recaudación tributaria municipal, en tanto que una reducción de la misma supone la disminución de ingresos con los cuales hacer frente a las necesidades derivadas del desarrollo de las políticas municipales que necesitan del gasto local.

Por lo que respecta a las tasas y las contribuciones especiales, la potestad tributaria local (autonomía local) tiene un valor absoluto, pues las Entidades Locales pueden establecerlas o no en su ámbito territorial. En el caso de las tasas que establezcan los entes locales - y según se disponga en la correspondiente Ordenanza Fiscal -, podrán regular la tarifa que señale la cantidad a pagar, estableciendo una cuantía fija o proporcional. Para ello, se deberán tener en cuenta criterios genéricos de capacidad económica de los sujetos obligados a satisfacerlas. Y así, la LRHL establece un catálogo de supuestos 
de utilización privativa o aprovechamiento especial del dominio público local y de prestación de servicios por parte del ente local que podrán ser prestados por el municipio estableciendo la exacción de una tasa, aunque existen una serie de servicios sobre cuya prestación las entidades locales no pueden establecer tasas, tales como el abastecimiento de agua, la limpieza y alumbrado de vías públicas, la protección civil o la enseñanza en los niveles de educación obligatoria. Estas potestades normativas tienen su claro reflejo en los ingresos obtenidos por los tributos locales. Además de los recursos tributarios, los entes locales pueden obtener ingresos a través de las transferencias del Estado o de otras instituciones, de su patrimonio o del recurso al endeudamiento. Por ello, en primer lugar, analizaremos los ingresos obtenidos por los Ayuntamientos en los últimos años (en tanto que son los entes locales de mayor importancia cuantitativa, pues agrupan más de las dos terceras partes del ingreso y del gasto local) y de ellos los que son de naturaleza tributaria. Así, si atendemos a la clasificación económica de los recursos mencionados en el Presupuesto de ingresos, su evolución en cuanto a la participación en el total en el período 2006-2014 se muestra en el siguiente cuadro. En el mismo, la elección del período viene motivada porque el año 2006 fue excepcionalmente bueno para los municipios españoles en cuanto a obtención de ingresos y, es a partir de este año, cuando se empiezan a notar los efectos de la crisis económica. Finalizamos el período en 2014 porque es el último año del cual podemos disponer de datos de liquidación presupuestaria.

En este período, los ingresos fiscales pasaron de suponer 20.428,3 miles de euros en 2006 a $23.844,1$ miles en 2014 , un crecimiento del 16,7 por cien, mientras que los gastos corrientes crecieron un 8,1 por cien en ese mismo período. De hecho, la autonomía financiera -entendida como la relación entre los ingresos tributarios y el total de ingresos- pasó del 48,7 por cien en 2006 al 58,7 por cien en 2014, un aumento de diez puntos porcentuales. Asimismo, la participación de cada uno de los capítulos presupuestarios muestra cómo la relación entre los ingresos tributarios y el total de ingresos de los Ayuntamientos ha ido creciendo a lo largo de estos años, tal y como se observa en el cuadro 1.

Los ingresos fiscales suponen alrededor del 80 por cien (con ciertos altibajos) de los ingresos tributarios incluidos en el presupuesto de ingresos en los capítulos uno (impuestos directos), dos (impuestos indirectos) y tres (tasas y otros ingresos). Sin embargo, esta cantidad también recoge otros conceptos incluidos en los capítulos presupuestarios mencionados, principalmente la participación en los tributos del Estado de los municipios del modelo de cesión, puesto que algunos municipios incluyen los ingresos procedente de la participación en los tributos del Estado en los capítulos uno y dos del Presupuesto de Ingresos mientras que otros los incluyen en el capítulo cuatro de ingresos, relativo a las transferencias corrientes, lo cual dificulta la realización del análisis comparado. Por ello, hemos calculado los ingresos fiscales ${ }^{2}$ puesto que se trata de ingresos obtenidos de forma coactiva

2.- Para el cálculo de los ingresos fiscales se han considerado los derechos reconocidos obtenidos por IBI, IVTM, IIVTNU, IAE, ICIO, el Impuesto sobre gastos suntuarios, la imposición especial aplicada en Canarias (AIE e IGIC) y las Tasas y otros ingresos, excluyendo los impuestos estatales que se han cedido a las entidades locales, como consecuencia de la aplicación del modelo de participación en tributos del Estado a los municipios mayores de 75.000 habitantes. También se excluyen los precios públicos. 


\section{Cuadro 1. Derechos reconocidos de los ayuntamientos. Años 2006-2014. Participación}

\begin{tabular}{|l|c|c|c|c|c|c|c|c|c|}
\hline AÑOS & $\mathbf{0 6}$ & $\mathbf{0 7}$ & $\mathbf{0 8}$ & $\mathbf{0 9}$ & $\mathbf{1 0}$ & $\mathbf{1 1}$ & $\mathbf{1 2}$ & $\mathbf{1 3}$ & $\mathbf{1 4}$ \\
\hline & & & & & & & & & \\
Imp. Directos: & 25,1 & 26,7 & 27,8 & 25,5 & 29,7 & 34,8 & 33,3 & 40,4 & 40,3 \\
IBI & 14,5 & 15,8 & 17,1 & 16,1 & 19,2 & 23,1 & 22,7 & 27,6 & 27,1 \\
IVTM & 4,3 & 4,5 & 4,5 & 4,0 & 4,4 & 4,9 & 4,4 & 4,9 & 4,6 \\
IIVTNU & 2,9 & 3,0 & 2,4 & 2,0 & 2,5 & 2,8 & 2,7 & 4,1 & 4,7 \\
IAE & 2,8 & 2,9 & 2,9 & 2,5 & 2,8 & 3,1 & 2,8 & 3,0 & 3,0 \\
Imp. Indirectos: & 6 & 5,5 & 4,2 & 2,6 & 2,5 & 2,6 & 1,9 & 2,2 & 2,1 \\
ICIO & 5,2 & 4,7 & 3,3 & 2,0 & 1,8 & 1,7 & 1,2 & 1,0 & 1,0 \\
Tasas y otros ing. & 17,6 & 17,3 & 17 & 14,6 & 16 & 17,8 & 15,6 & 16,9 & 16,2 \\
Ingresos Fiscales & 40,1 & 41,3 & 40,4 & 35,5 & 38,3 & 47,4 & 44,1 & 51,8 & 51,3 \\
ING. TRIBUTARIOS & 48,7 & 49,5 & 49 & 42,7 & 48,2 & 55,2 & 50,8 & 59,5 & 58,6 \\
Transf. corrientes & 27,1 & 29,9 & 30,9 & 26,6 & 27 & 30,8 & 26,8 & 30,1 & 29 \\
Ing. Pat. & 3,4 & 2,7 & 3 & 2,2 & 2,3 & 2,7 & 2 & 2,1 & 2,4 \\
OP. CORR. & 79,2 & 82,1 & 82,8 & 71,6 & 77,4 & 88,7 & 79,6 & 91,8 & 90,1 \\
OP. CAPITAL & 14,4 & 12 & 10,2 & 19,6 & 15,9 & 7,4 & 3,7 & 3,5 & 4 \\
OP.NO FINANC & 93,6 & 94,1 & 93 & 91,1 & 93,4 & 96,1 & 83,2 & 95,3 & 94,1 \\
OP. FINANC. & 6,4 & 5,9 & 7 & 8,9 & 6,6 & 3,9 & 16,8 & 4,7 & 5,9 \\
TOTAL ING. & 100 & 100 & 100 & 100 & 100 & 100 & 100 & 100 & 100 \\
\hline
\end{tabular}

FUENTE: Elaboración propia a partir de datos del MINHAP (Ministerio de Hacienda y Administraciones Públicas): http://www.minhafp.gob.es.

por parte de la Administración local. Por otro lado, el conocimiento de la evolución de los ingresos tributarios también resulta de interés y, por ello, hemos procedido al análisis de ambos conceptos.

El Impuesto sobre Bienes Inmuebles, IBI, es el impuesto que mayor aportación realiza al Presupuesto municipal, lo cual, desde una perspectiva de la fijación de criterios normativos para la descentralización fiscal, resulta lo más idóneo para financiar las haciendas locales (Poveda y Sánchez, 2002). El crecimiento de participación del IBI en los tributos que recaudan los Ayuntamientos ha sido importante en los últimos años. Así, en 2006 suponía el 35,4 por cien de los ingresos fiscales y en 2014 han pasado a suponer el 53,7 por cien. Es decir, ante la caída del ICIO, los Ayuntamientos han conseguido una mayor recaudación por IBI. En datos absolutos, en 2006 el Presupuesto definitivo de los Ayuntamientos por IBI era de 7.420.285 miles de euros y en 2014 de 12.797 .131 miles de euros, casi el doble. 
Por tanto, ante el gran descalabro sufrido por los Ayuntamientos como consecuencia de la reducción de la actividad urbanística y de los ingresos asociados a la misma - relacionados con el ICIO, el IIVTNU y determinadas tasas-, las entidades locales han optado por incrementar la recaudación por IBI. A ello, ha ayudado sin duda las sucesivas modificaciones incorporadas por el Estado en la normativa, tanto en lo referido al aumento de los tipos impositivos como de la base imponible.

El incremento de la participación de los ingresos tributarios de diez puntos desde el principio hasta el final de período analizado ha supuesto que los Ayuntamientos hayan incrementado considerablemente su autofinanciación, si bien se obtiene principalmente como consecuencia de la reducción de la participación de las operaciones de capital en la misma cuantía, aunque con distinto signo. Esta reducción ha venido motivada por la crisis económica y la disminución de los ingresos procedentes de la enajenación de inversiones reales y de transferencias de capital por parte del Estado (excepto el año 2009, que con el Plan E aprobado por el Gobierno, se incrementaron considerablemente las transferencias de capital del Estado a las entidades locales, si bien este fue un hecho aislado y puntual).

Un elemento a considerar es la participación del capítulo referido a tasas y otros ingresos en la financiación municipal. En este capítulo, son las Tasas principalmente las que aportan la mayor parte de los ingresos que se recogen. Y su evolución, con alguna excepción, ha sido creciente a lo largo de los últimos años. Ello ha sido debido, principalmente, a la necesidad de incrementar los recursos locales con unas bases imponibles que han ido aumentando por motivaciones diversas, tales como el desarrollo urbanístico o la aparición de nuevos objetos de gravamen relacionados con la tecnología de la información y la comunicación (Suárez y Fernández, 2008).

La participación de la recaudación de los tributos sobre el total es un dato importante a considerar para poder realizar un acercamiento a la realidad de la financiación local. Sin embargo, puede ser complementada con la información referida a los valores por habitante, pues puede ser representativa de las diferencias entre municipios en atención al estrato de población en el que se encuentren. Al análisis de esta cuestión nos dedicamos en el epígrafe siguiente, así como al estudio de la evolución de la eficacia de la gestión recaudatoria y del esfuerzo fiscal.

\section{Recaudación tributaria y crisis. Factores determinantes de la recaudación fiscal}

En este contexto de crisis económica y de necesidad por parte de los Ayuntamientos de incrementar los ingresos tributarios, es interesante analizar la evolución, en términos de ingresos fiscales por habitante o presión fiscal local, que se muestra a continuación. La presión fiscal se define tradicionalmente como la relación entre la recaudación y el PIB (Poveda y Sánchez, 2002; Pérez, 2006) 
y esta es la magnitud que se suele calcular de manera oficial cuando se obtienen datos de presión fiscal. Otros autores la definen como la relación entre los ingresos fiscales y la renta familiar disponible (Cárcaba, 2003) y en otros casos se define como la relación entre los impuestos y el número de habitantes (Zafra y López, 2006; Benito et al, 2010). Este indicador, que relaciona la recaudación con el número de habitantes, también ha sido definido como la capacidad fiscal potencial (Vallés y Zárate, 2013), en tanto que comparamos los ingresos por habitante por tramo de población con el total.

Esta magnitud se ha incrementado para todos los municipios españoles desde el principio hasta el final del período 2006-2014, tal y como observamos en el cuadro 2:

\section{Cuadro 2. Ingresos fiscales por habitante y por tramos de población. 2006-2014}

\begin{tabular}{|l|c|c|c|c|c|c|c|c|c|c|}
\hline $\begin{array}{l}\text { ESTRATOS } \\
\text { DE POB. } \\
\text { (miles htes) }\end{array}$ & $\mathbf{2 0 0 6}$ & $\mathbf{2 0 0 7}$ & $\mathbf{2 0 0 8}$ & $\mathbf{2 0 0 9}$ & $\mathbf{2 0 1 0}$ & $\mathbf{2 0 1 1}$ & $\mathbf{2 0 1 2}$ & $\mathbf{2 0 1 3}$ & $\mathbf{2 0 1 4}$ & $\begin{array}{c}\text { CTO } \\
\mathbf{2 0 1 4 - 2 0 0 6}\end{array}$ \\
\hline$>\mathbf{1 . 0 0 0}$ & 613,6 & 657,6 & 672,9 & 735,2 & 749,2 & 752,4 & 783,2 & 852,2 & 909,7 & 296,1 \\
$\mathbf{5 0 0}$ a 1.000 & 459,4 & 477,4 & 497,8 & 512,9 & 513,3 & 516,4 & 537,1 & 538,6 & 554,4 & 94,9 \\
$\mathbf{1 0 0}$ a $\mathbf{5 0 0}$ & 485,2 & 508,1 & 497,2 & 501,4 & 524,3 & 521,4 & 533,2 & 498,4 & 521,8 & 36,7 \\
$\mathbf{5 0}$ a $\mathbf{1 0 0}$ & 571,2 & 574,9 & 569,8 & 568,5 & 594,5 & 591,0 & 609,5 & 613,3 & 644,2 & 73,0 \\
$\mathbf{2 0}$ a $\mathbf{5 0}$ & 550,3 & 539,2 & 529,4 & 532,1 & 546,3 & 546,7 & 568,3 & 552,7 & 569,0 & 18,6 \\
$\mathbf{5}$ a $\mathbf{2 0}$ & 541,1 & 522,8 & 513,0 & 496,1 & 517,9 & 516,3 & 540,3 & 510,3 & 525,5 & $-15,6$ \\
$<=\mathbf{5}$ & 468,1 & 468,6 & 455,7 & 451,7 & 467,4 & 469,5 & 492,6 & 475,0 & 497,6 & 29,5 \\
\hline TOTAL & $\mathbf{5 2 5 , 7}$ & $\mathbf{5 3 2 , 2}$ & $\mathbf{5 2 7 , 1}$ & $\mathbf{5 3 2 , 2}$ & $\mathbf{5 5 0 , 8}$ & $\mathbf{5 5 0 , 0}$ & $\mathbf{5 7 0 , 3}$ & $\mathbf{5 5 9 , 3}$ & $\mathbf{5 8 3 , 9}$ & $\mathbf{5 8 , 1}$ \\
\hline
\end{tabular}

FUENTE: MINHAP (Ministerio de Hacienda y Administraciones Públicas) (ed): "HHLL en cifras" (varios años).

Los municipios han aumentado desde el principio hasta el final del período los ingresos fiscales por habitante, siendo Madrid y Barcelona -los únicos que se encuentran en el estrato de más de 1.000.000 de habitantes- los que en mayor medida han incrementado la recaudación por habitante 0 presión fiscal. Sin embargo, en un análisis más desagregado, los municipios con población comprendida entre 5.001 y 20.000 habitantes han reducido los ingresos fiscales por habitante en 2014 con respecto a las magnitudes obtenidas en el año 2006.

Los municipios de menos de 5.000 habitantes, que son casi el 84 por cien de los municipios españoles y agrupan alrededor del 13 por cien de la población, mantienen unos niveles de presión fiscal inferiores al resto de Ayuntamientos y esta situación se mantiene a lo largo del período considerado.

La recaudación por habitante o presión fiscal, no tiene en cuenta el nivel de renta, por lo que esto puede suponer que los municipios con mayor tamaño puedan tener mayores valores de la magnitud 
analizada, puesto que se está obviando el hecho del nivel de renta más elevado de sus ciudadanos (Cárcaba, 2003, Benito et al, 2010; Vallés y Zárate, 2013). Sin embargo, no debemos olvidar que las diferencias entre ingresos fiscales por habitante y por tramos de población pueden estar directamente relacionadas con la evolución de los impuestos locales a lo largo de los últimos años por cada tramo de población. Por ello, en el Cuadro 3, nos dedicaremos al análisis de cada uno de estos tributos y su evolución durante los años de crisis económica. Para ello, el índice de ingresos fiscales por habitante y por tipo de impuesto en 2006 y en 2014 es un indicador adecuado que analizamos en el cuadro siguiente:

\section{Cuadro 3. Índice de ingresos fiscales por habitante y por tipo de impuesto. 2006 y 2014}

\begin{tabular}{|c|c|c|c|c|c|c|c|c|c|c|}
\hline \multirow[b]{2}{*}{ MILES HTES } & \multicolumn{2}{|c|}{ IBI } & \multicolumn{2}{|c|}{ IVTM } & \multicolumn{2}{|c|}{ IIVTNU } & \multicolumn{2}{|c|}{ IAE } & \multicolumn{2}{|c|}{ ICIO } \\
\hline & 2006 & 2014 & 2006 & 2014 & 2006 & 2014 & 2006 & 2014 & 2006 & 2014 \\
\hline$>1.000$ & 121,3 & 144,9 & 105,6 & 101,8 & 193,2 & 327,0 & 142,9 & 148,5 & 60,6 & 181,9 \\
\hline $500-1.000$ & 103,8 & 89,9 & 98,1 & 99,2 & 101,4 & 113,6 & 104,3 & 126,0 & 51,6 & 82,1 \\
\hline $100-500$ & 96,4 & 87,4 & 101,1 & 96,5 & 103,6 & 76,6 & 117,9 & 108,3 & 71,0 & 87,5 \\
\hline $50-100$ & 114,8 & 114,8 & 102,6 & 103,6 & 124,9 & 119,5 & 97,4 & 108,6 & 104,5 & 88,5 \\
\hline 20-50 & 105,9 & 100,2 & 102,3 & 101,3 & 102,3 & 79,4 & 94,5 & 82,1 & 109,4 & 89,0 \\
\hline 5- 20 & 94,3 & 94,3 & 98,6 & 100,0 & 72,7 & 56,8 & 74,9 & 76,4 & 136,8 & 85,9 \\
\hline$<=5$ & 76,7 & 85,7 & 91,8 & 100,2 & 35,2 & 25,4 & 77,8 & 83,0 & 135,6 & 111,9 \\
\hline TOTAL & 100,0 & 100,0 & 100,0 & 100,0 & 100,0 & 100,0 & 100,0 & 100,0 & 100,0 & 100,0 \\
\hline
\end{tabular}

FUENTE: MINHAP (Ministerio de Hacienda y Administraciones Públicas) (ed): "HHLL en cifras" (varios años).

El análisis del índice de ingresos fiscales por habitante y por tipo de impuesto puede ser revelador para conocer la estructura de los recursos tributarios de los municipios y su evolución. En el IBI, son los municipios de más de 1.000 .000 de habitantes los que han incrementado en mayor medida el índice calculado desde el principio hasta el final del período. También lo incrementan en casi diez puntos los municipios de menos de 5.000 habitantes, mientras que en los municipios entre 500.000 y 1.000.000 de habitantes se produce una caída de alrededor de catorce puntos. En esta situación, la evolución de la recaudación por el IBI según tramos de población ha sido distinta. En conjunto, el crecimiento medio de la recaudación ha sido del 7,6 por cien, pero mientras en los municipios de más de un millón de habitantes el crecimiento medio ha sido del 9,5 por cien, en los de 5000.000 a 1.000 .000 de habitantes ha sido del 5,1 por cien. Es decir, en el período 2006 - 2014, el crecimiento de la recaudación por encima de la media se produce en los municipios de más de 1.000 .000 de habitantes, en los que tienen una población entre 50.000 y 100.000 habitantes y en los de menos de 5.000 habitantes. En el resto, el crecimiento medio de la recaudación está por debajo de la obtenida para el con- 
junto nacional. Por lo tanto, no todos los municipios han reaccionado al mismo ritmo de crecimiento en la recaudación del IBI, existiendo diferencias según el tamaño de la población.

En el IVTM, las diferencias son menores entre los diferentes tramos de población, siendo los Ayuntamientos con una población menor de 5.000 habitantes los que en mayor medida han aumentado el índice desde el principio hasta el final del período. Por el contrario, los municipios con más de 1.000.000 de habitantes lo han reducido.

En el IIVTNU resulta muy significativa la diferencia entre números índice al principio y al final del período para Madrid y Barcelona - que son las ciudades comprendidas en el grupo de municipios mayores de 1.000 .000 de habitantes-, pues se incrementa de manera notable y también para los municipios entre 500.000 y 1.000 .000 de habitantes. Mientras, para el resto de municipios el índice obtenido en 2014 es inferior al de 2006. Por lo tanto, en este tributo, son los municipios mayores los que han conseguido un crecimiento del índice analizado a lo largo del período, mientras que en los municipios de menos de 500.000 habitantes se ha reducido dicho valor y esto puede estar relacionado con el objeto de gravamen del impuesto. En efecto, este tributo grava el incremento del valor del terreno de naturaleza urbana que se pone de manifiesto como consecuencia de la transmisión de bienes inmuebles de naturaleza urbana y es más posible que en los municipios más grandes se hayan producido más transmisiones en su conjunto y de mayor cuantía que en el resto.

En el IAE la recaudación por habitante apenas varía desde el principio hasta el final del período, si bien se reduce en los municipios entre 20.000 y 50.000 habitantes (en 3,8 euros por habitante) y en los municipios entre 100.000 y 500.000 habitantes (en 2,9 euros). En el resto, se mantiene en los mismos valores a lo largo de estos años, excepto en los municipios entre 500.000 y 1.000 .000 de habitantes, que es donde más crece, lo cual se traduce en el mayor crecimiento del índice analizado con respecto al resto de municipios.

En el ICIO la recaudación por habitante se ha reducido en casi 49 euros desde 2006 hasta 2014, pues ha pasado de suponer 59,2 euros por habitante en 2006 a solamente 10,4 en 2014, como consecuencia de la crisis de la construcción, en tanto que se trata de un tributo que grava la instalación, construcción u obra para la cual se necesite una licencia de obras. En este contexto, la mayor reducción se produce en los municipios más pequeños. Así, tienen una reducción por encima de la media todos los municipios inferiores a 100.000 habitantes, lo cual explica los valores del índice tan elevados para los municipios de Madrid y Barcelona, puesto que estos Ayuntamientos han visto reducir la recaudación por ICIO por habitante en 17 euros desde el principio hasta el final del período. Un análisis de la evolución de las tasas medias y de la variación de la recaudación por habitante - realizada en el Cuadro 4 - puede ayudar a mejorar el análisis de la situación: 


\section{Cuadro 4. Impuestos locales. Tasas medias de crecimiento y variación de la recaudación por habitante entre 2006 y 2014}

\begin{tabular}{|c|c|c|c|c|c|c|c|c|c|c|}
\hline \multirow[b]{2}{*}{ MILES HTES } & \multicolumn{2}{|c|}{ IBI } & \multicolumn{2}{|c|}{ IVTM } & \multicolumn{2}{|c|}{ IIVTNU } & \multicolumn{2}{|c|}{ IAE } & \multicolumn{2}{|c|}{ ICIO } \\
\hline & $\operatorname{Tm}(\%)$ & $\Delta \mathrm{Rec} / \mathrm{h}$ & $\operatorname{Tm}(\%)$ & $\Delta \mathrm{Rec} / \mathrm{h}$ & $\operatorname{Tm}(\%)$ & $\Delta \operatorname{Rec} / \mathrm{h}$ & $\operatorname{Tm}(\%)$ & $\Delta \operatorname{Rec} / \mathrm{h}$ & $\operatorname{Tm}(\%)$ & $\Delta \operatorname{Rec} / \mathrm{h}$ \\
\hline$>1.000$ & 9,5 & 210,1 & $-0,5$ & $-2,4$ & 14,5 & 97,3 & 0,6 & 1,9 & $-6,9$ & $-17,0$ \\
\hline $500-1.000$ & 5,1 & 83,0 & 0,0 & 0,0 & 8,4 & 22,5 & 2,5 & 7,0 & $-13,2$ & $-22,0$ \\
\hline $100-500$ & 6,4 & 88,2 & 0,1 & $-2,7$ & 2,5 & 3,5 & $-0,2$ & $-2,9$ & $-15,2$ & $-33,0$ \\
\hline $50-100$ & 8,4 & 135,3 & 1,4 & $-0,1$ & 6,6 & 17,6 & 3,0 & 3,6 & $-18,7$ & $-52,7$ \\
\hline 20- 50 & 7,7 & 108,8 & 1,1 & $-1,1$ & 4,5 & 5,3 & $-0,3$ & $-3,8$ & $-20,0$ & $-55,5$ \\
\hline $5-20$ & 7,3 & 111,3 & 0,4 & 0,1 & 2,8 & 4,0 & 0,8 & 0,5 & $-22,9$ & $-72,0$ \\
\hline$<=5$ & 8,1 & 115,9 & 0,7 & 3,6 & 1,5 & 0,9 & 0,9 & 1,7 & $-20,7$ & $-68,6$ \\
\hline TOTAL & 7,6 & 117,9 & 0,4 & $-0,6$ & 6,5 & 16,2 & 0,7 & 0,1 & $-18,5$ & $-48,8$ \\
\hline
\end{tabular}

FUENTE: MINHAP (Ministerio de Hacienda y Administraciones Públicas) (ed): "HHLL en cifras" (varios años).

El mayor crecimiento de la tasa media durante el período 2006-2014 para el aumento de los ingresos se ha centrado en el IBI y, en segundo lugar, a gran distancia el IIVTNU. En el caso del IBI se ha visto fuertemente influenciado por las subidas realizadas en los últimos años. Baste citar para ello el incremento de los tipos impositivos para 2012 y para 2013 - contenidos en el Real Decreto-ley 20/2011, de 30 de diciembre, de medidas urgentes en materia presupuestaria, tributaria y financiera para la corrección del déficit público - que posteriormente fue prorrogado para 2014 y 2015. Por lo tanto, el aumento que se produjo en la recaudación por IBI no está solamente relacionada con la política fiscal municipal, pues fue el Estado el que decidió aumentar los ingresos de los municipios realizando estas modificaciones en el IBI, utilizando las potestades normativas que tiene asignadas. Las diferencias entre tramos de población son importantes, puesto que mientras los ciudadanos que residen en los municipios de más de 1.000 .000 de habitantes vieron crecer sus pagos en 210 euros desde 2006 hasta 2014, los habitantes de municipios entre 500.001 y 1.000 .000 de habitantes solamente tuvieron que aumentar sus aportaciones a las arcas locales en 83 euros. Las desigualdades más importantes se obtienen en 2014 entre los municipios con más población y los que menos tienen. Así, en los municipios de más de 1.000 .000 los ingresos por habitante correspondientes al IBI ascendieron a 411,3 euros, mientras que en los municipios de menos de 5.000 habitantes, dicha cantidad ascendía a 243,2 euros, una diferencia apreciable que supone una presión fiscal mucho menor por este tributo en los municipios pequeños.

El IIVTNU se vio afectado por la crisis económica y, en los primeros años del período analizado, su recaudación fue disminuyendo año tras año. Sin embargo, en 2013 y 2014 aumentaron signifi- 
cativamente sus ingresos con respecto a las cifras de años anteriores. A ello pudo contribuir las modificaciones realizadas en este tributo por la Ley 16/2013, que modificó diferentes aspectos del tributo. No obstante, en la actualidad, el Tribunal Constitucional se ha pronunciado en 2017, tanto en los territorios forales como en el resto del territorio nacional, y se han declarado inconstitucionales determinados preceptos de la normativa del tributo. En esencia, los contribuyentes que vendieron su vivienda con pérdidas tuvieron que abonar el IIVTNU, en tanto que la base imponible se fija en función el valor catastral y un coeficiente determinado según el número de años transcurridos desde la transmisión anterior, cifras que no contemplan el valor de transmisión y de adquisición. El pronunciamiento constitucional ha supuesto la solicitud, en numerosos casos, de los ingresos abonados indebidamente y no prescritos a las arcas locales, con el consiguiente efecto en la recaudación impositiva local. La necesidad de una solución y, por tanto, una modificación de la regulación del tributo podría llevar al establecimiento de un supuesto de no sujeción al IIVTNU en aquellos casos en los cuales se realice una transmisión de un bien inmueble de naturaleza urbana y se obtengan pérdidas. En cualquier caso, los efectos recaudatorios en el tributo son evidentes y se pondrán de manifiesto en las cifras presupuestarias.

Otras variables relacionadas con la recaudación tributaria local son la referida a la eficacia de la gestión recaudatoria y el esfuerzo fiscal. Al análisis de ambas nos dedicamos a continuación.

\subsection{La eficacia de la gestión recaudatoria}

Un aspecto que. sin duda, ha influido en los ingresos tributarios obtenidos por los municipios es el relacionado con la gestión tributaria municipal, pues es esencial para el cumplimiento del Presupuesto planteado por el ente local, el cual tiene su contraprestación en un determinado nivel de gasto público.

Para ello, podemos realizar un análisis del indicador de la eficacia de la gestión recaudatoria, $E_{g r}$, - o índice de recaudación, como lo denominan Cárcaba (2003) o Morala y Fernández (2006)- que viene definido como:

$$
E_{g r}=(\text { Recaudación / Derechos reconocidos }) \times 100
$$

En efecto, los derechos reconocidos ofrecen las cantidades que existe compromiso de cobro, pero el dinero que efectivamente ha entrado en caja vendría determinado por la recaudación obtenida. En la medida que los derechos reconocidos se acerquen a la recaudación, la gestión recaudatoria será mejor (Pont, 2001; Alonso, 2002: Lago, 2003). Lo ideal sería que coincidieran al cien por cien, si bien pueden existir diferentes motivos por los cuales no se correspondan; por ejemplo, si hay compromisos de cobro que finalmente se convierten en insolvencias.

En el período 2006-2014, la eficacia de la gestión recaudatoria la podemos analizar en el Cuadro 5: 


\section{Cuadro 5. Eficacia de la gestión recaudatoria. 2006 y 2014}

\begin{tabular}{|l|cc|cc|cc|cc|cc|}
\hline \multirow{2}{*}{ MILES HTES } & \multicolumn{2}{|c|}{ IBI } & \multicolumn{2}{c|}{ IVTM } & \multicolumn{2}{c|}{ IIVTNU } & \multicolumn{2}{c|}{ IAE } & \multicolumn{2}{c|}{ ICIO } \\
& $\mathbf{2 0 0 6}$ & $\mathbf{2 0 1 4}$ & $\mathbf{2 0 0 6}$ & $\mathbf{2 0 1 4}$ & $\mathbf{2 0 0 6}$ & $\mathbf{2 0 1 4}$ & $\mathbf{2 0 0 6}$ & $\mathbf{2 0 1 4}$ & $\mathbf{2 0 0 6}$ & $\mathbf{2 0 1 4}$ \\
\hline \multirow{3}{*}{1.000} & 92,4 & 91,7 & 83,5 & 79,2 & 92,5 & 88,9 & 88,1 & 88,7 & 87,8 & 81,6 \\
$500-1.000$ & 89,6 & 77,0 & 83,9 & 80,2 & 91,1 & 75,3 & 92,1 & 78,8 & 80,2 & 59,4 \\
$100-500$ & 88,1 & 86,4 & 87,2 & 84,0 & 85,3 & 75,5 & 86,2 & 83,5 & 81,8 & 66,3 \\
$50-100$ & 88,8 & 86,8 & 86,9 & 83,3 & 84,2 & 73,8 & 88,8 & 87,9 & 85,7 & 72,4 \\
$20-50$ & 89,4 & 85,4 & 88,0 & 83,5 & 80,3 & 67,3 & 90,7 & 87,9 & 84,6 & 74,5 \\
$5-20$ & 87,4 & 84,7 & 87,8 & 84,9 & 79,0 & 68,7 & 87,3 & 88,4 & 85,4 & 81,6 \\
$<=5$ & 88,7 & 86,0 & 88,9 & 87,7 & 77,1 & 72,5 & 88,2 & 92,9 & 87,1 & 88,9 \\
& & & & & & & & & & \\
\hline TOTAL & $\mathbf{8 8 , 8}$ & $\mathbf{8 6 , 2}$ & $\mathbf{8 7 , 0}$ & $\mathbf{8 3 , 8}$ & $\mathbf{8 4 , 9}$ & $\mathbf{7 7 , 9}$ & $\mathbf{8 8 , 2}$ & $\mathbf{8 6 , 8}$ & $\mathbf{8 5 , 0}$ & $\mathbf{7 6 , 2}$ \\
\hline
\end{tabular}

FUENTE: MINHAP (Ministerio de Hacienda y Administraciones Públicas) (ed): "HHLL en cifras" (varios años).

Por impuestos, el IBI, el IAE y el IVTM son los que presentan una mayor eficacia de la gestión recaudatoria (coincidiendo con los impuestos de carácter obligatorio), lo cual probablemente estará relacionado con el hecho de gestionarse a través de padrones con los cuales el municipio dispone de todos los datos del sujeto pasivo. El ICIO, que presenta valores del 85 por cien en 2006 reduce considerablemente la eficacia de la gestión recaudatoria conforme avanza la crisis urbanística y, en los últimos años - que ha caído considerablemente la recaudación por este tributo-, este indicador se ha reducido en 9 puntos porcentuales, siendo especialmente significativa la reducción en los municipios con población entre 20.000 y 1.000 .000 de habitantes.

Cabría esperar que la eficacia de la gestión recaudatoria hubiera aumentado con el transcurrir de los años, en tanto que los municipios disponen de mayores y mejores medios para el control tributario municipal. Sin embargo, para todos los impuestos, los resultados de este indicador son menores al final que al principio del período, lo cual sería una cuestión a considerar por parte del gestor local. En el IBI, resulta destacable la reducción del indicador analizado en los municipios con población entre 500.000 y 1.000 .000 de habitantes, que han visto reducir la eficacia de la gestión recaudatoria del tributo en alrededor de doce puntos porcentuales, lo cual tiene difícil explicación en tanto que, en principio, al ser más grandes, dispondrán de mayores medios materiales y humanos para mejorar la gestión tributaria que los Ayuntamientos con menor población.

En el caso del IIVTNU, la reducción del indicador es de casi siete puntos y es posible que en los próximos años, fruto de la inconstitucionalidad de determinados preceptos del tributo, se reduzca todavía más. 
Por tanto, en 2014, los municipios de Madrid y Barcelona son los que presentan un mayor valor del indicador de la eficacia de la gestión recaudatoria en el IBI, mientras que el IVTM presenta, curiosamente, un mayor valor de indicador en los municipios de menos de 5.000 habitantes, que son los que disponen de menos medios para desarrollar esta tarea y lo mismo ocurre para el IAE y el ICIO. Esto también podría reflejar que los municipios pequeños han depurado mejor su cifra de derechos reconocidos, mientras que el resto pueden tener un mayor porcentaje de derechos pendientes de cobro al final del año.

En cualquier caso, a la vista de los resultados analizados, resulta evidente la necesidad de la mejora de la eficacia de la gestión recaudatoria por parte de todos los municipios, con las particularidades por impuestos que hemos ido analizando.

Junto a la eficacia de la gestión recaudatoria, otro elemento que podemos considerar es el referido a la variable esfuerzo fiscal, puesto que puede servir de elemento explicativo de la evolución de la recaudación en dichos municipios. A su análisis procedemos en el apartado siguiente.

\subsection{El esfuerzo fiscal}

El esfuerzo fiscal puede ser analizado desde dos perspectivas. Por un lado, el cálculo que se realiza por parte del Estado para la obtención del valor de la variable esfuerzo fiscal, aplicable a los municipios del modelo de variables. Por otro, el cálculo que se ha realizado por parte de diversos autores (Suárez y Fernández, 2008; Vallés y Zárate, 2013 o Poveda y Sánchez, 2002) para conocer el esfuerzo fiscal realizado por los municipios.

En el primer caso, en el marco de la financiación municipal, los Ayuntamientos reciben una participación en los ingresos del Estado. Este sistema de participación distingue dos modelos: a) el modelo de cesión, que resulta aplicable a los municipios que tengan una población de derecho igual o superior a 75.000 habitantes 0 que sean capitales de provincia, o de comunidad autónoma, a los cuales se les cede un porcentaje de determinados impuestos estatales, y b) el modelo de variables, aplicable al resto de municipios. Asimismo, los municipios turísticos gozan de ciertas particularidades en cuanto a la aplicación de los mencionados modelos. En 2013, el número de municipios integrados en el modelo de cesión ascendía a 97, los cuales agrupaban al 44 por cien de la población, mientras que 8.021 municipios se encontraban incluidos en el modelo de variables, aglutinando al 56 por cien restante. Para determinar la participación en los ingresos del Estado en el modelo de variables, una vez que se calcula la participación total que corresponde a todos los municipios, se distribuye entre todos ellos atendiendo a una serie de criterios, tales como el número de habitantes (el 75 por 100 del total), el esfuerzo fiscal (el 12,5 por 100 del total), y el inverso de la capacidad tributaria (el 12,5 por 100 del total). 
El esfuerzo fiscal anual se define en la Ley de Presupuestos Generales del Estado para cada año y para cada impuesto obligatorio (es decir, Impuesto sobre Bienes Inmuebles, Impuesto sobre Actividades económicas e Impuesto sobre Vehículos de tracción mecánica) con una fórmula que relaciona la recaudación real y la potencial máxima.

El inverso de la capacidad tributaria relaciona la base imponible media por habitante del municipio con la de su estrato poblacional referida al IBI. Desde 2006 hasta 2013 la financiación resultante de la aplicación de las variables esfuerzo fiscal e inverso de la capacidad tributaria ha evolucionado tal y como se observa en el Gráfico 1:

\section{Gráfico 1. Evolución de la financiación de las variables esfuerzo fiscal e inverso de la capacidad tributaria. Millones de euros. Años 2006-2013}

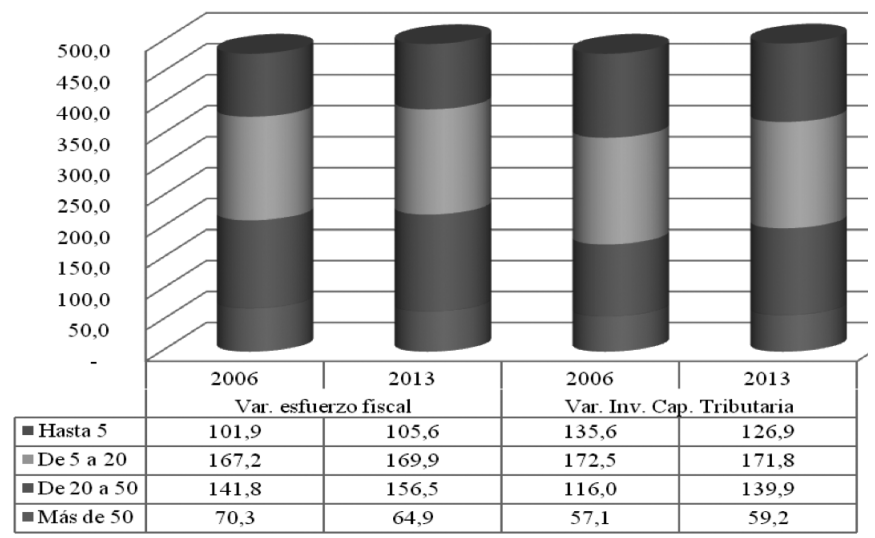

FUENTE: MINHAP (Ministerio de Hacienda y Administraciones Públicas) (ed): "HHLL en cifras". Años 2006 y 2013.

En los municipios de entre 20.000 y 50.000 habitantes es en los que más ha crecido la financiación proveniente del esfuerzo fiscal y del inverso de la capacidad tributaria. Por el contrario, los municipios de más de 50.000 habitantes presentan una tasa de crecimiento negativa, lo cual está relacionado con sus posibilidades de obtener, o no, unos mayores ingresos.

Tras el análisis de estas variables que se refieren, como ya hemos comentado en líneas anteriores, solamente a los municipios integrados en el modelo de variables, hemos querido conocer el esfuerzo fiscal para el conjunto de los Ayuntamientos mediante una simulación que nos pueda llevar a la obtención del cálculo aproximado del mismo. Las dificultades que plantea el cálculo del esfuerzo fiscal han sido expuestas por Suárez y Fernández (2008), analizando las distintas fórmulas para el 
cálculo del mismo. Vallés y Zárate, (2013) calculan el esfuerzo fiscal de los municipios aragoneses utilizando un enfoque de frontera estocástica. Poveda y Sánchez (2002) por su parte, calculan el esfuerzo fiscal mediante el cociente entre el tipo impositivo fijado por el Gobierno local y el tipo máximo establecido en la LRHL. Sin embargo, una de las cuestiones en las cuales coinciden los diversos autores es la relativa a la consideración de la definición de esfuerzo fiscal como la relación entre recaudación real y potencial y, además, esta es la definición general que se define en los Presupuestos Generales del Estado para el cálculo de la variable esfuerzo fiscal en el modelo de variables. Desde una aproximación al cálculo del esfuerzo fiscal, la recaudación real podemos obtenerla de las cifras de recaudación, mientras que la potencial podríamos calcularla. Si aceptamos que la base por el tipo puede ser una aproximación de la cuota y la suma de las cuotas, la recaudación y partimos de los tipos impositivos fijados por los Ayuntamientos, -disponibles en la página web del Ministerio de Hacienda y Administraciones Públicas-, podemos calcular la base, en tanto que dispondríamos de la recaudación y del tipo. Una vez conseguida la base y multiplicada por los tipos máximos de cada tributo, podríamos calcular la recaudación potencial. Es decir:

$$
\underline{\mathrm{R}}=\text { Base } \times \zeta
$$

siendo $\underline{R}$, la recaudación real, Base, la base imponible global total de cada tributo y $\zeta$ los tipos impositivos de los diferentes tributos según tramo de población. Por otro lado, debemos considerar:

$$
\overline{\mathrm{R}}=\text { Base } \times \underline{\mathrm{I}}
$$

siendo $\bar{R}$ la recaudación potencial, Base, la base imponible total y $\mathrm{I}$ el tipo impositivo máximo para cada tributo que fija la LRHL.

Quedando, por tanto, el esfuerzo fiscal, $\varepsilon$, definido como:

$$
\varepsilon=(\underline{R} / \bar{R}) \times 100
$$

Para el cálculo del esfuerzo fiscal hemos procedido del modo siguiente, según los diferentes impuestos locales. En primer lugar, hemos utilizado los tipos del IBI de urbana, en tanto que es el que mayor aportación realiza a la recaudación por este tributo. En lo referido al IVTM, hemos considerado el coeficiente por tramo de tamaño para los turismos, puesto que es el tipo de vehículo que genera la mayor parte de la recaudación (Poveda y Sánchez, 2002). En el IIVTNU, hemos considerado la media del tipo impositivo por tramos de población en atención al número de años transcurridos desde la anterior transmisión. En el IAE solamente disponemos del coeficiente de situación mínimo y máximo, por lo que hemos calculado la media. Por último, en el ICIO disponíamos del tipo impositivo por cada tramo de población. 
Para el cálculo del esfuerzo fiscal por tramos de población, hemos optado por la clasificación que distingue según lo que se denomina como "tramo de tamaño fiscal del municipio", que es, en esencia, una agrupación de los municipios por tramos de población distinta a la habitual y utilizada hasta ahora en el presente trabajo. Hemos usado esta clasificación porque es la que aparece en la desagregación de los tipos impositivos locales en los datos utilizados del Ministerio de Hacienda y acorde con el estudio que pretendemos realizar. Somos conscientes de que este cálculo no considera variables que se han tenido en cuenta en otros trabajos y, además, no contempla la existencia de bonificaciones y deducciones que disminuyen el tipo efectivo, pero puede ser un indicador aproximado de este indicador.

Los resultados obtenidos se muestran a continuación. En este caso, el esfuerzo fiscal de los municipios españoles por impuestos y por tramos de población en 2006 y 2014 aparece en el Cuadro 6:

\section{Cuadro 6. Esfuerzo fiscal de los municipios españoles por tramos de población. 2006 y 2014. Porcentajes}

\begin{tabular}{|l|cc|cc|cc|cc|cc|}
\hline \multirow{2}{*}{ Mil. htes/año } & \multicolumn{2}{|c|}{ IBI } & \multicolumn{2}{c|}{ IVTM } & \multicolumn{2}{c|}{ IIVTNU } & \multicolumn{2}{c|}{ IAE } & \multicolumn{2}{c|}{ ICIO } \\
& $\mathbf{2 0 0 6}$ & $\mathbf{2 0 1 4}$ & $\mathbf{2 0 0 6}$ & $\mathbf{2 0 1 4}$ & $\mathbf{2 0 0 6}$ & $\mathbf{2 0 1 4}$ & $\mathbf{2 0 0 6}$ & $\mathbf{2 0 1 4}$ & $\mathbf{2 0 0 6}$ & $\mathbf{2 0 1 4}$ \\
\hline \multirow{2}{*}{ Hasta 5 } & 53,5 & 56,7 & 61,0 & 58,3 & 73,7 & 71,6 & 29,6 & 28,9 & 61,8 & 62,0 \\
$5-20$ & 60,5 & 59,8 & 73,7 & 77,5 & 84,1 & 82,3 & 37,5 & 40,9 & 75,8 & 78,5 \\
$20-50$ & 63,3 & 63,8 & 83,1 & 85,5 & 88,5 & 86,0 & 45,0 & 47,9 & 85,8 & 87,5 \\
$50-100$ & 67,3 & 63,6 & 83,3 & 88,0 & 91,9 & 87,6 & 48,9 & 57,9 & 89,3 & 89,5 \\
Más 100 & 60,1 & 63,4 & 89,5 & 94,3 & 94,2 & 91,3 & 53,6 & 61,1 & 93,3 & 95,0 \\
& & & & & & & & & & \\
\hline TOTAL & $\mathbf{6 0 , 6}$ & $\mathbf{5 7 , 4}$ & $\mathbf{8 0 , 8}$ & $\mathbf{6 1 , 8}$ & $\mathbf{8 9 , 3}$ & $\mathbf{7 5 , 6}$ & $\mathbf{4 5 , 3}$ & $\mathbf{3 1 , 4}$ & $\mathbf{8 4 , 3}$ & $\mathbf{6 5 , 5}$ \\
\hline
\end{tabular}

FUENTE: Elaboración propia a partir de datos de recaudación y tipos del Ministerio de Hacienda y Administraciones Públicas. En: http://www.minhafp.gob.es.

Los resultados conseguidos están en la línea de los obtenidos por Poveda y Sánchez (2002) para 1999, excepto en lo referido al IAE.

Desde 2006 hasta 2014 los municipios han reducido su esfuerzo fiscal en todos los impuestos, lo cual contrasta con las necesidades financieras del período. Por tanto, los Ayuntamientos españoles todavía pueden incrementar su recaudación de manera notable en algunos impuestos, tales como el IBI 0 el IAE. En el caso del IBI, a pesar del aumento de tipos de los últimos años, todavía queda un margen importante para el incremento de la recaudación. En el IAE, las diferencias que se sufren en el mismo por parte de los contribuyentes como consecuencia de las modificaciones realizadas en 
el tributo en 2004, que han generado graves problemas de equidad, hacen que el argumento de elevar la recaudación por este impuesto sea más complejo, máxime si tenemos en cuenta que el Estado compensa a los municipios por la pérdida recaudatoria del IAE y en este trabajo no hemos considerado estas cantidades.

Por tramos de población, existen diferencias notables. Así, son los municipios de más de 100.000 habitantes los que han aumentado en casi todos los tributos su esfuerzo fiscal, si bien es necesario considerar que son también los de mayor renta. En cualquier caso, no podemos encontrar un patrón de comportamiento de todos los municipios, pues mientras los de población entre 50.000 y 100.000 habitantes han incrementado su esfuerzo fiscal en el IAE en 8,9 puntos desde el principio hasta el final del período, los Ayuntamientos de menos de 5.000 habitantes han reducido su esfuerzo fiscal. Y lo mismo ocurre en otros casos e impuestos.

No obstante, el hecho de que los Ayuntamientos españoles puedan incrementar su esfuerzo fiscal, principalmente en lo referido al IBI y, al resto de tributos, no implica forzosamente la necesidad de incrementar su recaudación (Bahl, 1971; Vallés y Zárate, 2013). Además, debemos considerar que algunos municipios pueden decidir exigir menos tributos porque consideran la realización de una política de gasto público más restrictiva (Cheliah et al, 1975, Vallés y Zárate, 2013).

Un aspecto que puede haber incidido es el referido al aumento en los últimos años del tipo impositivo del IBI, lo cual incide en la recaudación potencial y el incremento de la misma, suponiendo un incremento del denominador del esfuerzo fiscal definido, lo cual puede haber tenido sus consecuencias en la obtención de las cifras de esfuerzo fiscal obtenidas.

En cualquier caso, tras el análisis realizado, consideramos de interés seguir avanzando en esta línea de investigación para trabajos futuros que puedan seguir aportando elementos que nos sirvan para un mayor análisis de la realidad tributaria local.

\section{Conclusiones}

Los municipios españoles han atravesado numerosas dificultades económicas derivadas de la crisis económica, como el resto de entidades públicas. Ante esta situación, los Ayuntamientos han optado por incrementar los recursos tributarios, aumentando así su autonomía financiera, principalmente a través del IBI, principal figura de la tributación local, siendo los municipios de más de 1.000 .000 de habitantes los que en mayor medida han incrementado los ingresos fiscales por habitante desde el principio hasta el final del período. 
Este crecimiento de la recaudación ha estado relacionado con el incremento de tipos del IBI, regulado en la norma estatal para todos los municipios, junto con el ejercicio de las potestades normativas de cada Ayuntamiento. Otros factores explicativos de la evolución de la recaudación tributaria están relacionados con la eficiencia de la gestión recaudatoria: En este sentido, es necesario resaltar que los municipios han reducido la eficacia de la gestión recaudatoria desde el principio hasta el final del período para todos los impuestos, principalmente en los de población comprendida entre 500.000 y 1.000.000 de habitantes, lo cual contrasta con el hecho de que los municipios con mayor población cuentan con más recursos materiales y humanos para la realización de la gestión tributaria.

Las principales reducciones de este indicador se han producido en los impuestos voluntarios, IIVTNU e ICIO, si bien en el caso del ICIO los derechos reconocidos se han reducido considerablemente desde el principio hasta el final del período, por lo que, en principio, se podría haber esperado una mejora del indicador. Las variables relacionadas con el esfuerzo fiscal y con el inverso de la capacidad tributaria para los municipios del modelo de variables, pueden ser indicadores de las variaciones en la recaudación y, en el período analizado, ha sido en los municipios entre 20.000 y 50.000 habitantes en los que más ha crecido la financiación por estas variables.

El cálculo del esfuerzo fiscal realizado pone de manifiesto la reducción del mismo en todos los impuestos desde 2006 hasta 2014, siendo el ICIO el que mayor reducción de este indicador presenta, relacionado, con toda probabilidad, con la reducción de la recaudación proveniente de este tributo como consecuencia de la crisis inmobiliaria. Sin embargo, por tramos de población la situación varía, pues en los Ayuntamientos de más de 100.000 habitantes se produce un incremento del esfuerzo fiscal en este período en todos los tributos excepto en el IIVTNU, probablemente como consecuencia de su mayor renta. Mientras, en otros tramos de población, la situación es diferente.

Los problemas derivados de la rigidez de los tributos locales sin duda han colaborado para la situación actual de la Hacienda Local, por lo que deberían ser objeto de modificación y atender a criterios que supusieran una mayor elasticidad de este tipo de tributación, lo cual podría suponer una aportación importante a la consecución de una mayor autofinanciación municipal.

En cualquier caso, esta línea de trabajo puede seguir siendo objeto de numerosos estudios que puedan profundizar en la relación entre la recaudación tributaria y otros indicadores que puedan añadir nuevas perspectivas al estudio de la situación tributaria local y su desarrollo (Fernández, Kapfer \& Creutzig, 2016, Bosch y Suarez, 2015), con nuevas líneas de reforma que se puedan plantear en el futuro, tales como las referidas a la cooperación entre municipios (Niaounakis and Blank, 2017), que puede reducir considerablemente el gasto local 0 , al menos, hacerlo más eficiente.

No obstante, los resultados obtenidos pueden ser de gran interés para el gestor local y para la toma de decisiones en su municipio, considerando cuál es la situación de los Ayuntamientos que se encuentran en su mismo tramo de población, sirviendo así al planteamiento de soluciones a los problemas que se analizan en el presente trabajo. 


\section{Bibliografía}

ALONSO, M. (2002): "La aplicación de los tributos locales. Relaciones entre la gestión tributaria y la inspección tributaria local", Gaceta Fiscal, 208, 95-106.

ANGOITIA, M. (2005): "Competencias y presupuesto de gastos de los municipios", Papeles y memorias de la Real academia de Ciencias Morales y políticas, XIII, 110-129.

BAHL, R. (1971): "A regression approach to tax effort and tax ratio analysis", IMF Staf Papers, 18 (3), 570-612.

BALAGUER-COLL, M.T., PRIOR, D. \& TORTOSA-AUSINA, E. (2016): "On the determinants of local government debt: Does zone size fit all?", International Public Management Journal, vol 19, (4), 513-542.

BENITO, B., BASTIDA, F. \& MUÑOZ, M.J. (2010): "Factores explicativos de la presión fiscal municipal", Revista de Contabilidad, 13 (2), 239-283.

BOSCH, N. \& SUÁREZ, J. (2015): "Politics and finance in spanish municipalities", Hacienda Pública Española, 212, 51-66.

CARAMES, L. (2005): "Consideraciones en torno al tamaño de los municipios", Papeles y Memorias de la Real Academia de Ciencias Morales y Políticas, 13, 40-45.

CARCABA, A. (2003): Análisis financiero de las entidades locales mediante el uso de indicadores. Revista Española de Financiación y Contabilidad, 118, 661-692.

CASTELLS, A. (2002): "Algunos comentarios sobre la reforma de las haciendas locales", Papeles de Economía Española, 92, 8-26.

CHELLIAH, R.J., BAAS, H.J. \& KELLY, M. (1975): "Tax ratios and tax effort in developing countries, 1969-1971", International Monetary Fund Papers, 22, 187-205.

FERNÁNDEZ, B., KAPFER, D. \& CREUTZIG, F. (2016): "A systematic framework of location value taxes reveals dismal policy design in most Euroepean countries", Lan Use Policy, 51, 335-349.

FORCADELL, X. (2015): "Balance de la reforma del régimen local en España un año después: ideas clave", Revista Democracia y Gobierno Local, 28, 5-10.

GUILLAMÓN, M.D., BENITO, B. \& BASTIDA, F. (2011): "Evaluación de la deuda pública local en España", Revista Española de Financiación y Contabilidad, 40, 251-285.

LAGO, J.M. (2003): "Tributos y procedimientos principios y normas generales. La colaboración entre el Estado y los entes locales en la gestión tributaria", Tributos Locales, 35, 25-40. 
MINISTERIO DE HACIENDA Y ADMINISTRACIONES PUBLICAS (ed): Haciendas Locales en cifras. Años 2006 a 2014.

MORALA, M.B. \& FERNÁNDEZ, J.M. (2006): "Análisis de entidades públicas mediante indicadores: Instrumento de rendición de cuentas y demostración de responsabilidades", Pecunia, 2, 79-100.

NIAOUNAKIS, T. \& BLANK, J. (2017): "Inter-municipal cooperation, economies of scale and cost efficiency: an application of stochastic frontier analysis to dutch municipal tax departments", Local Government Studies, 43 (4), 533-554.

PEDRAJA, F. \& SUÁREZ, J. (2011): "Crisis y reforma de la hacienda local: ¿hay alguien por ahí?", Economistas, 29, 186-190.

PONT, J.F. (2001): "La necesidad de establecer un nuevo modelo de gestión de los recursos financieros locales: gestión de los tributos". En: Pont Mestres, M. (coord.), Financiación de los entes locales, Marcial Pons, Valencia, 369-410.

POVEDA, F. \& SÁNCHEZ, A. (2002): "La financiación impositiva municipal. Propuestas para su reforma". En: IX Encuentro de Economía Pública, Vigo.

SUÁREZ, J. (2001): "El futuro de la financiación local en el contexto del Estado de las Autonomías", Hacienda Pública Española, número extra 2001, 359-377.

SUÁREZ, J. (2005): "La nueva financiación local: ¿un modelo permanente?”. En: Medina, M. y Arroyo, A., Las haciendas locales: situación actual y líneas de reforma, Fundación Democracia y Gobierno Local, 117-138.

SUÁREZ, J. (2007): "Insuficiencia relativa, gastos impropios y financiación local", Papeles de Economía Española, 113, 241-252.

SUÁREZ, J. y FERNÁNDEZ, R. (2008): "Esfuerzo fiscal municipal y aportación del usuario al coste de los servicios". En: XV Encuentro de Economía Pública, Salamanca.

SUÁREZ, J. et al. (2008): La financiación local en España: radiografía del presente y propuestas de futuro, FEMP, Madrid.

SUÁREZ, J. \& FERNÁNDEZ, R. (2012): "Impactos de la crisis sobre la Hacienda local española: datos y retos básicos". En: Portillo, M.J. \& Merino, V., Crisis económica y entidades locales, Lex Nova, Valladolid, 59-92.

TRANCHEZ, J.M., et al. (2011): "Los gastos no obligatorios en los municipios de Madrid, Cataluña y Andalucía", Documentos de Trabajo del Instituto de Estudios Fiscales, 25, 1-39.

VALLES, J. \& ZARATE, A. ("013): "Esfuerzo fiscal de los municipios aragoneses desde un enfoque de frontera estocástica". En: XX Encuentro de Economía Pública, Sevilla.

ZAFRA, J.L. \& LÓPEZ, A.M. (2006): "La valoración de la condición financiera de los ayuntamientos andaluces", Auditoría Pública, 39, 41-52. 